\title{
CLUSTER SET THEOREMS FOR UNIFORMLY CONVERGENT SEQUENCES OF FUNCTIONS
}

\author{
PETER COLWELL
}

1. Introduction. Let $f(z)$ be a complex-valued function defined in $D:\{|z|<1\}$, with values on the Riemann sphere $S$. At any point $e^{i \theta}$ of $C:\{|z|=1\}$, the (interior) cluster set, $C_{D}\left(f, e^{i \theta}\right)$, is defined as follows: $\alpha \in C_{D}\left(f, e^{i \theta}\right)$ if there exists a sequence $\left\{z_{n}\right\}$ in $D$ such that $\lim _{n \rightarrow \infty} z_{n}=e^{i \theta}$ while $\lim _{n \rightarrow \infty} f\left(z_{n}\right)=\alpha$. For any point $e^{i \theta}$ of $C, C_{D}\left(f, e^{i \theta}\right)$ is closed and nonempty. If $G$ is a subset of $D$ whose closure contains $e^{i \theta}$, the partial cluster set, $C_{G}\left(f, e^{i \theta}\right)$, is defined analogously by requiring the sequence $\left\{z_{n}\right\}$ to lie in $G$. (For a more detailed introduction to the theory of cluster sets, see [1].)

In this paper we consider a sequence $\left\{f_{n}(z)\right\}$ of functions defined in $D$ and converging uniformly to a function $f(z)$ in $D$. In $\$ 2$ we consider the convergence of a sequence of cluster sets for $\left\{f_{n}(z)\right\}$ at $e^{i \theta}$ to the corresponding cluster set for $f(z)$ at $e^{i \theta}$. The function $f(z)$ is said to have an ambiguous point at $e^{i \theta}$ if there exist two simple arcs, $K$ and $L$, in $D$ terminating at $e^{i \theta}$ for which $C_{K}\left(f, e_{i \theta}\right) \cap C_{L}\left(f, e^{i \theta}\right)=\varnothing$. (The original references and statements about ambiguous points may be found in $[1$, p. 39].) In $\$ 3$ we relate the ambiguous points of $f(z)$ to those of $\left\{f_{n}(z)\right\}$. In $\$ 4$ we present related results for mappings from an arbitrary topological space into a compact metric space.

Let $Z$ be a compact metric space with metric $d$. For any nonempty closed subset $A$ of $Z$ and any $\epsilon>0$, we let $A+\epsilon=\{z \in Z: \exists a \in A$ with $d(a, z)<\epsilon\}$. If $\left\{A_{n}\right\}$ is a sequence of nonempty closed subsets of $Z$, we say $\lim _{n \rightarrow \infty} A_{n}=A$ if for any $\epsilon>0$ there exists an integer $N$ such that $A_{n} \subset A+\epsilon$ and $A \subset A_{n}+\epsilon$ whenever $n>N$. It is in this sense that we discuss the convergence of a sequence of cluster sets.

2. Our hypothesis in the following is that $\left\{f_{n}(z)\right\}$ is a sequence of arbitrary complex-valued functions converging uniformly in $D$ to a function $f(z)$. For simplicity, we shall denote by $|a-b|$ the distance between $a$ and $b$ on $S$ in the spherical metric.

TheOREM 1. If $e^{i \theta}$ is any point of $C$, then $\lim _{n \rightarrow \infty} C_{D}\left(f_{n}, e^{i \theta}\right)=C_{D}\left(f, e^{i \theta}\right)$, and this convergence is uniform in $e^{i \theta}$.

Proof. Let $e^{i \theta}$ be an arbitrary point of $C$, and let $\epsilon>0$ be arbitrarily chosen. Then for some positive integer $N$, whenever $n>N$ and $z \in D$, $\left|f_{n}(z)-f(z)\right|<\epsilon / 3$.

Received by the editors May 20, 1966. 
Choose any $n>N$, and select any point $\alpha_{n}$ from $C_{D}\left(f_{n}, e^{i \theta}\right)$. Then there exists a sequence $\left\{a_{k}(n)\right\} \subset D$ such that $\lim _{k \rightarrow \infty} a_{k}(n)=e^{i \theta}$ and $\lim _{i \rightarrow \infty} f_{n}\left[a_{k}(n)\right]=\alpha_{n}$ For some subsequence $\left\{a_{j}(n)\right\}$ of $\left\{a_{i}(n)\right\}$ there exists $\lim _{j \rightarrow \infty} f\left[a_{j}(n)\right]=w$, where $w \in C_{D}\left(f, e^{i \theta}\right)$, while $\lim _{j \rightarrow \infty} f_{n}\left[a_{j}(n)\right]$ $=\alpha_{n}$. Now we can find an integer $J$ such that whenever $j>J$, both $\left|f\left[a_{j}(n)\right]-w\right|<\epsilon / 3$ and $\left|f_{n}\left[a_{j}(n)\right]-\alpha_{n}\right|<\epsilon / 3$. But then $\left|\alpha_{n}-w\right|$ $\leqq\left|\alpha_{n}-f_{n}\left[a_{j}(n)\right]\right|+\left|f_{n}\left[a_{j}(n)\right]-f\left[a_{j}(n)\right]\right|+\left|f\left[a_{j}(n)\right]-w\right|<\epsilon$ whenever $j>J$, so that for $\alpha_{n} \in C_{D}\left(f_{n}, e^{i \theta}\right), n>N$, we have $\alpha_{n} \in C_{D}\left(f, e^{i \theta}\right)+\epsilon$.

Now let any $w \in C_{D}\left(f, e^{i \theta}\right)$ be chosen. Then there exists a sequence $\left\{a_{k}\right\} \subset D$ with $\lim _{k \rightarrow \infty} a_{k}=e^{i \theta}$ and $\lim _{k \rightarrow \infty} f\left(a_{k}\right)=w$. For each $n>N$ we can select a subsequence $\left\{a_{k}(n)\right\}$ of $\left\{a_{k}\right\}$ along which $f_{n}(z)$ has a limit $\alpha_{n} \in C_{D}\left(f_{n}, e^{i \theta}\right)$, while $\lim _{k \rightarrow \infty} f\left[a_{k}(n)\right]=w$. A repetition of the argument above will show that if $w \in C_{D}\left(f, e^{i \theta}\right)$, then $w \in C_{D}\left(f_{n}, e^{i \theta}\right)+\epsilon$ for each $n>N$.

By our definition, $\lim _{n \rightarrow \infty} C_{D}\left(f_{n}, e^{i \theta}\right)=C_{D}\left(f, e^{i \theta}\right)$, and since $N$ above is independent of the point $e^{i \theta}$, this convergence is uniform in $e^{i \theta}$.

An obvious modification of the proof of Theorem 1 yields

TheOREM 2. Let $G$ be any subset of $D$ whose closure contains a point $e^{i \theta}$ of $C$. Then $\lim _{n \rightarrow \infty} C_{G}\left(f_{n}, e^{i \theta}\right)=C_{G}\left(f, e^{i \theta}\right)$. In particular, if $G$ is the radius, $\rho$, to $e^{i \theta}, \lim _{n \rightarrow \infty} C_{\rho}\left(f_{n}, e^{i \theta}\right)=C_{\rho}\left(f, e^{i \theta}\right)$.

From Theorem 2 we may state the following

Corollary. If $L$ is any simple arc in $D$ terminating at $e^{i \theta}$ on $C$, then $f(z)$ has a limit $\gamma$ as $z$ approaches $e^{i \theta}$ along $L$ if, and only if, $\lim _{n \rightarrow \infty} C_{L}\left(f_{n}, e^{i \theta}\right)=\{\gamma\}$. In particular, $f(z)$ has a radial limit $\lim _{r \rightarrow 1} f\left(r e^{i \theta}\right)$ $=\gamma$ if, and only if, $\lim _{n \rightarrow \infty} C_{\rho}\left(f_{n}, e^{i \theta}\right)=\{\gamma\}$.

A point $\alpha$ belongs to the boundary cluster set, $C_{B}\left(f, e^{i \theta}\right)$, for a function $f(z)$ at $e^{i \theta}$ if there exist: (i) a sequence $\left\{\tau_{k}\right\}$ of points on $C-\left\{e^{i \theta}\right\}$ such that $\lim _{k \rightarrow \infty} \tau_{k}=e^{i \theta}$ : and (ii) a sequence of points $\left\{\omega_{k}\right\}$ with $\omega_{k} \in C_{D}\left(f, \tau_{k}\right)$ such that $\lim _{k \rightarrow \infty} \omega_{k}=\alpha$. If in (ii) we require that $\omega_{k} \in C_{\rho}\left(f, \tau_{k}\right)$, the radial cluster set of $f(z)$ at $\tau_{k}$, then we have the definition of the radial boundary cluster set, $C_{B R}\left(f, e^{i \theta}\right)$, for $f(z)$ at $e^{i \theta}$. (For details of the role these cluster sets play in the boundary behavior of functions meromorphic in $D$, see [1] and the paper of W. B. Woolf [3].)

Theorem 3. For any point $e^{i \theta}$ on $C, \lim _{n \rightarrow \infty} C_{B}\left(f_{n}, e^{i \theta}\right)=C_{B}\left(f, e^{i \theta}\right)$, and $\lim _{n \rightarrow \infty} C_{B R}\left(f_{n}, e^{i \theta}\right)=C_{B R}\left(f, e^{i \theta}\right)$.

Proof. It suffices to prove the first of these statements. Let any $\epsilon>0$ be given; for some integer $N,\left|f_{n}(z)-f(z)\right|<\epsilon / 9$ for all $z$ in $D$ whenever $n>N$. Choose any integer $n>N$, and let $\alpha(n)$ be an arbi- 
trary point of $C_{B}\left(f_{n}, e^{i \theta}\right)$. Then there exists a sequence $\left\{\tau_{k}(n)\right\}$ on $C-\left\{e^{i \theta}\right\}$ and a sequence $\left\{\omega_{k}(n)\right\}$ such that $\lim _{k \rightarrow \infty} \tau_{k}(n)=e^{i \theta}, \omega_{k}(n)$ $\in C_{D}\left(f_{n}, \tau_{k}\right)$, and $\lim _{k \rightarrow \infty} \omega_{k}(n)=\alpha(n)$.

From the proof of Theorem 1 we have $C_{D}\left(f_{n}, \tau\right) \subset C_{D}(f, \tau)+\epsilon / 3$ for $n>N$ and any $\tau \in C$; thus for each value of $k$ there exists a point $\gamma_{k}(n) \in C_{D}\left(f, \tau_{k}\right)$ such that $\left|\omega_{k}(n)-\gamma_{k}(n)\right|<\epsilon / 3$. From the sequence $\left\{\gamma_{k}(n)\right\}$ we can select a convergent subsequence-which for simplicity we denote by $\left\{\gamma_{k}(n)\right\}$ itself-with a limit $\gamma \in C_{B}\left(f, e^{i \theta}\right)$. There exists an integer $K(\epsilon, n)$ such that $\left|\omega_{k}(n)-\alpha(n)\right|<\epsilon / 3$ and $\left|\gamma_{k}(n)-\gamma\right|$ $<\epsilon / 3$ whenever $k>K(\epsilon, n)$. Then for any $k>K(\epsilon, n)$ we may write $|\alpha(n)-\gamma| \leqq\left|\alpha(n)-\omega_{k}(n)\right|+\left|\omega_{k}(n)-\gamma_{k}(n)\right|+\left|\gamma_{k}(n)-\gamma\right|<\epsilon$, so that $\alpha(n) \in C_{B}\left(f, e^{i \theta}\right)+\epsilon$, or $C_{B}\left(f_{n}, e^{i \theta}\right) \subset C_{B}\left(f, e^{i \theta}\right)+\epsilon$ for $n>N$.

We wish to show also that for $n>N C_{B}\left(f, e^{i \theta}\right) \subset C_{B}\left(f_{n}, e^{i \theta}\right)+\epsilon$. Let $\alpha$ be an arbitrary point of $C_{B}\left(f, e^{i \theta}\right)$. Then for a sequence $\left\{\tau_{k}\right\}$ on $C-\left\{e^{i \theta}\right\}$ with $\lim _{k \rightarrow \infty} \tau_{k}=e^{i \theta}$ there is a sequence $\left\{\omega_{k}\right\}$ such that $\omega_{k} \in C_{D}\left(f, \tau_{k}\right)$ and $\lim _{k \rightarrow \infty} \omega_{k}=\alpha$.

For $n>N$ and any $\tau \in C$ we have $C_{D}(f, \tau) \subset C_{D}\left(f_{n}, \tau\right)+\epsilon / 3$. Thus for fixed $n>N$ and each $k$ we may select a point $\gamma_{k}(n) \in C_{D}\left(f_{n}, \tau_{k}\right)$ such that $\left|\omega_{k}-\gamma_{k}(n)\right|<\epsilon / 3$. From this point on the argument repeats that above to show that $C_{B}\left(f, e^{i \theta}\right) \subset C_{B}\left(f_{n}, e^{i \theta}\right)+\epsilon$ for $n>N$. Hence $\lim _{n \rightarrow \infty} C_{B}\left(f_{n}, e^{i \theta}\right)=C_{B}\left(f, e^{i \theta}\right)$ for any point $e^{i \theta}$ on $C$.

Similar statements of convergence can be made in terms of other types of cluster sets at a point on $C$.

3. Ambiguous points. If $\left\{f_{n}(z)\right\}$ converges uniformly in $D$ to $f(z)$, it is an easy consequence of Theorem 2 that each ambiguous point of $f(z)$ on $C$ is an ambiguous point for all but finitely many functions $f_{n}(z)$. Thus a function defined in $D$ having an ambiguous point on $C$ cannot be uniformly approximated in $D$ by functions having no ambiguous points.

However, the limit of a uniformly convergent sequence of functions, each with an ambiguous point on $C$, need not have an ambiguous point. As a simple example, define a sequence $\left\{f_{n}(z)\right\}$, where $f_{n}(z)=z$ for $z \in D-K-L, f_{n}(z)=e^{i \theta}+1 / n$ for $z \in K, f_{n}(z)=e^{i \theta}-1 / n$ for $z \in L$, where $n=1,2,3, \cdots$ and $K, L$ are simple arcs in $D$ terminating at $e^{i \theta}$ on $C$. The sequence converges uniformly in $D$ to a function $f(z)$, with $f(z)=z$ for $z \in D-K-L, f(z)=e^{i \theta}$ for $z \in K \cup L$. For each $n$, $e^{i \theta}$ is an ambiguous point of $f_{n}(z)$, but $f(z)$ has no ambiguous points.

If we assign a crude measure to the extent to which a point of $C$ is ambiguous for a function in $D$, we can relate the points which are "uniformly" ambiguous for the uniformly convergent sequence $\left\{f_{n}(z)\right\}$ and the ambiguous points of their limit $f(z)$. Let us say that 
$f(z)$ is $\delta$-ambiguous at $e^{i \theta}$ if there exist simple arcs $K$ and $L$ in $D$ terminating at $e^{i \theta}$ such that for each $\alpha \in C_{K}\left(f, e^{i \theta}\right)$ and each $\beta \in C_{L}\left(f, e^{i \theta}\right)$, $|\alpha-\beta| \geqq \delta>0$.

THEOREM 4. If for all $n$ and some $\delta>0 f_{n}(z)$ is $\delta$-ambiguous at $e^{i \theta}$, then $f(z)$ is ambiguous at $e^{i \theta}$.

Proof. Let $\rho$ be chosen, $0<\rho<\delta$, and let $\epsilon$ be chosen, $0<\epsilon<\delta-\rho$. For some integer $N$ and all $z$ in $D,\left|f_{n}(z)-f(z)\right|<\epsilon / 4$ when $n>N$. Select any $n>N$. For this $n$ there exist simple arcs, $K=K(n)$ and $L=L(n)$, in $D$ terminating at $e^{i \theta}$ such that $\left|\alpha_{n}-\beta_{n}\right| \geqq \delta$ for all $\alpha_{n} \in C_{K}\left(f_{n}, e^{i \theta}\right), \beta_{n} \in C_{L}\left(f_{n}, e^{i \theta}\right)$.

Let $\alpha, \beta$ be arbitrarily chosen from $C_{K}\left(f, e^{i \theta}\right), C_{L}\left(f, e^{i \theta}\right)$, respectively. We show that $|\alpha-\beta| \geqq \rho$. For some sequences $\left\{a_{j}\right\} \subset K$, $\left\{b_{j}\right\} \subset L$, we have $\lim _{j \rightarrow \infty} f\left(a_{j}\right)=\alpha, \lim _{j \rightarrow \infty} f\left(b_{j}\right)=\beta$. From these sequences we can select subsequences $\left\{a_{j}^{\prime}\right\},\left\{b_{j}^{\prime}\right\}$ such that $\lim _{j \rightarrow \infty} f_{n}\left(a_{j}^{\prime}\right)$ $=\gamma \in C_{K}\left(f_{n}, e^{i \theta}\right), \lim _{j \rightarrow \infty} f\left(a_{j}^{\prime}\right)=\alpha$, and $\lim _{j \rightarrow \infty} f_{n}\left(b_{j}^{\prime}\right)=\lambda \in C_{L}\left(f_{n}, e^{i \theta}\right)$, $\lim _{j \rightarrow \infty} f\left(b_{j}^{\prime}\right)=\beta$.

We can find an integer $J$ such that for $j>J,\left|f_{n}\left(a_{j}^{\prime}\right)-\gamma\right|<\epsilon / 8$, $\left|f_{n}\left(b_{j}^{\prime}\right)-\lambda\right|<\epsilon / 8,\left|f\left(a_{j}^{\prime}\right)-\alpha\right|<\epsilon / 8,\left|f\left(b_{j}^{\prime}\right)-\beta\right|<\epsilon / 8$. Then for $j>J:|\alpha-\gamma| \leqq\left|\alpha-f\left(a_{j}^{\prime}\right)\right|+\left|f\left(a_{j}^{\prime}\right)-f_{n}\left(a_{j}^{\prime}\right)\right|+\left|f_{n}\left(a_{j}^{\prime}\right)-\gamma\right|<\epsilon / 2$; and $|\beta-\lambda| \leqq\left|\beta-f\left(b_{j}^{\prime}\right)\right|+\left|f\left(b_{j}^{\prime}\right)-f_{n}\left(b_{j}^{\prime}\right)\right|+\left|f_{n}\left(b_{j}^{\prime}\right)-\lambda\right|<\epsilon / 2$. Now $|\lambda-\gamma| \geqq \delta$ by hypothesis, so $\delta \leqq|\lambda-\gamma| \leqq|\lambda-\beta|+|\beta-\alpha|$ $+|\alpha-\gamma|<\epsilon+|\alpha-\beta|<(\delta-\rho)+|\alpha-\beta|$, and $|\alpha-\beta|>\rho$. Consequently, $f(z)$ is ambiguous at $e^{i \theta}$.

4. Let $X$ be an arbitrary topological space and $Z$ be a compact metric space with metric $\rho$. For each $x \in X$ denote by $\mathfrak{u}_{x}$ the collection of open sets in $X$ containing $x$. For any nonempty subset $T$ of $X$ let $f$ be any mapping of $T$ into $Z$. J. D. Weston [2] defined the cluster set of $f$ at a point $t \in T$ to be $C(f ; t)=\Omega^{*} \mathrm{Cl}[f(U)]$, where $\mathrm{N}^{*}$ represents the intersection over all $U \in \mathfrak{U}_{t}$, and $\mathrm{Cl}(A)$ denotes the closure of $A$. For any $f$ mapping $T$ into $Z$ and any $t \in T$, we see that $C(f ; t)$ is nonempty and closed.

We state for reference the following lemma [2, p. 436].

Lemma. Let $t \in T$ and $K$ be a compact set in $Z$. Suppose that, corresponding to each $U \in \mathcal{U}_{t}$, a closed set $F(U)$ in $Z$ is prescribed so that: (i) if $U_{1} \subset U_{2}$, then $F\left(U_{1}\right) \subset F\left(U_{2}\right)$; (ii) $K \cap\left[\cap^{*} F(U)\right]=\varnothing$. Then there exists at least one $U \in \mathcal{U}_{t}$ such that $K \cap F(U)=\varnothing$.

Let $\left\{f_{n}\right\}$ be a sequence of mappings of $T$ into $Z$ which converges uniformly on $T$ to a mapping $f$. That is, given any $\epsilon>0$ there exists in teger $N$ such that whenever $n>N, \rho\left[f_{n}(t), f(t)\right]<\epsilon$ for all $t \in T$. 
Theorem 5. For any point $t \in T, \lim _{n \rightarrow \infty} C\left(f_{n} ; t\right)=C(f ; t)$, and this convergence is uniform in $t$.

Proof. Let $\epsilon>0$ be given. Then for some integer $N$, whenever $n>N, \rho\left[f_{n}(t), f(t)\right]<\epsilon / 4$ for all $t \in T$. Choose any $s \in T$; suppose for some integer $n>N$ that there exists $\alpha_{n} \in C\left(f_{n} ; s\right)$ such that $\rho\left(\alpha_{n}, \alpha\right) \geqq \epsilon$ for all $\alpha \in C(f ; s)$.

Let $K=\left\{z \in Z: \rho\left(\alpha_{n}, z\right) \leqq \epsilon / 2\right\} ; K$ is compact and $K \cap C(f ; s)=\varnothing$. Using the lemma with $F(U)=\mathrm{Cl}[f(U)]$, we have $K \cap \mathrm{Cl}[f(U)]=\varnothing$ for some $U \in \mathcal{U}_{s}$. For each $t \in U, \rho\left[\alpha_{n}, f(t)\right] \geqq \epsilon / 2$. But since $\alpha_{n} \in C\left(f_{n} ; s\right)$, $\alpha_{n} \in \mathrm{Cl}\left[f_{n}(U)\right]$, and we can find some $t^{\prime} \in U$ with $f_{n}\left(t^{\prime}\right) \neq \alpha_{n}$ and $\rho\left[\alpha_{n}, f_{n}\left(t^{\prime}\right)\right]<\epsilon / 4$. Now $\epsilon / 2 \leqq \rho\left[\alpha_{n}, f\left(t^{\prime}\right)\right] \leqq \rho\left[\alpha_{n}, f_{n}\left(t^{\prime}\right)\right]+\rho\left[f_{n}\left(t^{\prime}\right), f\left(t^{\prime}\right)\right]$ $<\epsilon / 2$. Thus for $n>N$ and any $\alpha_{n} \in C\left(f_{n} ; s\right)$, there must be some $\alpha \in C(f ; s)$ such that $\rho\left(\alpha_{n}, \alpha\right)<\epsilon$, and for $n>N$ and any $t \in T$ we have $C\left(f_{n} ; t\right) \subset C(f ; t)+\epsilon$.

Now choose any $s \in T$ and suppose there exists $n>N$ and $\alpha \in C(f ; s)$ for which $\rho\left(\alpha, \alpha_{n}\right) \geqq \epsilon$ for any $\alpha_{n} \in C\left(f_{n} ; s\right)$. If $K=\{z \in Z: \rho(\alpha, z) \leqq \epsilon / 2\}$ and $F(U)=\mathrm{Cl}[f(U)]$, then $K \cap C\left(f_{n} ; s\right)=\varnothing$, and the Lemma gives us a set $U \in \mathcal{u}_{s}$ for which $K \cap \mathrm{Cl}[f(U)]=\varnothing$. Hence for each $t \in U$, $\rho\left[\alpha, f_{n}(t)\right] \geqq \epsilon / 2$. Since $\alpha \in C(f ; s)$, we can find $t^{\prime} \in U$ with $f\left(t^{\prime}\right) \neq \alpha$, $\rho\left[f\left(t^{\prime}\right), \alpha\right]<\epsilon / 4$, and again we have $\epsilon / 2 \leqq \rho\left[f_{n}\left(t^{\prime}\right), \alpha\right] \leqq \rho\left[f_{n}\left(t^{\prime}\right), f\left(t^{\prime}\right)\right]$ $+\rho\left[f\left(t^{\prime}\right), \alpha\right]<\epsilon / 2$. Thus for any $n>N$ and any $\alpha \in C(f ; s)$, there exists $\alpha_{n} \in C\left(f_{n} ; s\right)$ such that $\rho\left(\alpha, \alpha_{n}\right)<\epsilon$, so that for $n>N$ and all $t \in T$, $C(f ; t) \subset C\left(f_{n} ; t\right)+\epsilon$.

Therefore, $\lim _{n \rightarrow \infty} C\left(f_{n} ; t\right)=C(f ; t)$, and since $N$ is independent of $t \in T$, this limit is uniform in $t$.

If $t$ is a point of $T$, let $A$ be any subset of $T$ such that $t \in \mathrm{Cl}(A)$. As a generalization of the boundary cluster set, Weston [2] defined the cluster set $C^{A}(f ; t)=\bigcap^{*}\{\mathrm{Cl}[M(f ; U ; A)]\}$, where for each $U \in \mathfrak{u}_{t}$ $M(f ; U ; A)=\bigcup_{A \cap U} C(f ; a)$. In addition, as a generalization of the partial cluster set, let $C_{A}(f ; t)=\bigcap^{*} \mathrm{Cl} f(A \cap U)$. Then simple modifications in the proof of Theorem 5 will yield

Theorem 6. $\lim _{n \rightarrow \infty} C^{A}\left(f_{n} ; t\right)=C^{A}(f ; t)$ and $\lim _{n \rightarrow \infty} C_{A}\left(f_{n} ; t\right)$ $=C_{A}(f ; t)$.

\section{BIBLIOGRAPHY}

1. K. Noshiro, Cluster sets, Springer, Berlin, 1960.

2. J. D. Weston, Some theorems on cluster sets, J. London Math. Soc. 33 (1958), 435-441.

3. W. B. Woolf, The boundary behavior of meromorphic functions, Ann. Acad. Sci. Fenn. A I No. 305 (1961) 1-11.

IOWA State University 